\title{
Classical double copy: Kerr-Schild-Kundt metrics from Yang-Mills theory
}

\author{
Metin Gürses ${ }^{1, *}$ and Bayram Tekin ${ }^{2, \dagger}$ \\ ${ }^{1}$ Department of Mathematics, Faculty of Sciences, Bilkent University, 06800 Ankara, Turkey \\ ${ }^{2}$ Department of Physics, Middle East Technical University, 06800 Ankara, Turkey
}

(Received 11 October 2018; published 28 December 2018)

\begin{abstract}
The classical double copy idea relates some solutions of Einstein's theory with those of gauge and scalar field theories. We study the Kerr-Schild-Kundt (KSK) class of metrics in $d$ dimensions in the context of possible new examples of this idea. We first show that it is possible to solve the Einstein-Yang-Mills system exactly using the solutions of a Klein-Gordon-type scalar equation when the metric is the $p p$-wave metric, which is the simplest member of the KSK class. In the more general KSK class, the solutions of a scalar equation also solve the Yang-Mills, Maxwell, and Einstein-Yang-Mills-Maxwell equations exactly, albeit with a null fluid source. Hence, in the general KSK class, the double copy correspondence is not as clearcut as in the case of the $p p$ wave. In our treatment, all the gauge fields couple to dynamical gravity and are not treated as test fields. We also briefly study Gödel-type metrics along the same lines.
\end{abstract}

DOI: 10.1103/PhysRevD.98.126017

\section{INTRODUCTION}

Constructing solutions of Einstein field equations, with a source or in a vacuum, is so difficult that anytime a new method is suggested, one should embrace it with enthusiasm. The recent "classical double copy" correspondence [1] is such a new idea, which we shall pursue here for some exact gravity waves in the hope of extending the earlier examples [2]. The basic essence of the classical double copy method is this: one can find some classical solutions of general relativity from the classical solutions of YangMills or Maxwell field equations or even from those of a simpler scalar field equation. This construction, gravity being a double copy of the YM theory-which itself is a single copy - and a scalar field (usually a biadjoint real scalar field) - which is the zeroth copy - is an extension of a powerful idea and observation that goes beyond the classical level: the scattering amplitudes in general relativity and those of two copies of Yang-Mills theories are related. This is known as the Bern-Carrasco-Johansson (BCJ) double copy [3] and works perturbatively, granted that the color and kinematic factors are identified accordingly. For more details and the references, see Refs. [4,5].

The classical double copy correspondence has been mostly studied in the Kerr-Schild class of metrics. This class has remarkable properties and includes a large number of physical metrics, including the Kerr black hole. In this work, to extend the set of examples and to understand the possible limitations to the classical double copy correspondence, we study a class of spacetime, the so-

*gurses@fen.bilkent.edu.tr †btekin@metu.edu.tr called Kerr-Schild-Kundt (KSK) class, which turned out to be universal, in the sense that KSK metrics solve all metricbased theories [6-11]. The classical double copy arguments make use of the metric in the Kerr-Schild form:

$$
g_{\mu \nu}=\bar{g}_{\mu \nu}+2 V \ell_{\mu} \ell_{\nu},
$$

where $\bar{g}_{\mu \nu}$ is the background (or the seed) metric; $\ell_{\mu}$ is a null vector with respect to both metrics; and $V$, at this stage, is an arbitrary function. The fact that $\ell_{\mu}$ is null is a crucial point in what follows. In fact, to see this explicitly, for the moment let us assume that it is not null. Then the inverse metric reads

$$
g^{\mu \nu}=\bar{g}^{\mu \nu}-\frac{2 V}{1+2 V \ell^{2}} \ell^{\mu} \ell^{\nu}
$$

where $\ell^{2} \equiv \bar{g}^{\mu \nu} \ell_{\mu} \ell_{\nu}$. It is clear that only for the null case, the inverse metric is linear in the metric profile function $V$, a fact that dramatically simplifies all the ensuing discussion. (Note that in the last part of this work, we briefly consider the metrics that are defined with a non-null vector field.)

In the works on classical double copies, one usually encounters the following construction: the seed metric is taken to be flat, namely $g_{\mu \nu}=\eta_{\mu \nu}+2 V \ell_{\mu} \ell_{\nu}$; the Maxwell and Yang-Mills fields are taken as $A_{\mu}=V \ell_{\mu}$; and the Yang-Mills field $\mathcal{A}_{\mu}^{a}=c^{a} V \ell_{\mu}$, where $c^{a}$ 's are constants. In this case, one can only treat the Maxwell and the YangMills as test fields. If the metric satisfies the vacuum field equations $\left(G_{\mu \nu}=0\right)$, then the spacetime becomes stationary, i.e., $\partial_{0} V=0[1,2]$, and the metric function satisfies Laplace's equation $\nabla^{2} V=0$. Any solution of this equation 
also solves the Maxwell and Yang-Mills equations identically. This construction was extended to the maximally symmetric nonflat backgrounds in Ref. [2]. If one relaxes the stationarity assumption, i.e., $\partial_{0} V \neq 0$, but instead imposes the constraint $\ell^{\mu} \partial_{\mu}=0$, one obtains a nice exact result which supports the classical double copy approach.

The layout of this work is as follows: we first start with the $p p$ waves and give a solution of the coupled EinsteinYang-Mills-Maxwell system that is in the double copy spirit. We then discuss a possible extension to the general KSK class and show that a null fluid is needed for that case for the correspondence to work. Our results are summarized in two theorems. In the conclusion and further discussions part, we also discuss a possible extension of these ideas to the Gödel-type metrics with non-null vector fields. The motivation is to extend the double copy correspondence possibly to the massive gauge field case.

\section{KSK METRICS AND DOUBLE COPY}

Our first main result is on the exact solutions of the Einstein-Yang-Mills-Maxwell field equations, where the spacetime is the $d$-dimensional $p p$-wave geometry. We first state our main results for the $p p$ waves as a theorem and provide the proof later as a subclass of the KSK case.

Setting all relevant coupling constants to unity, the coupled Einstein, Maxwell, and Yang-Mills equations are

$$
\begin{aligned}
G_{\mu \nu}= & \gamma_{a b} \mathcal{F}^{a \alpha_{\mu}} \mathcal{F}_{\alpha \nu}^{b}-\frac{1}{4} \mathcal{F}^{2} g_{\mu \nu} \\
& +\sum_{k=1}^{N}\left(F^{k \alpha}{ }_{\mu} F_{\alpha \nu}^{k}-\frac{1}{4} F^{k^{2}} g_{\mu \nu}\right), \\
\nabla_{\mu} F^{k \mu \nu}= & 0,\left(D_{\mu} \mathcal{F}^{\mu \nu}\right)^{a}=0,
\end{aligned}
$$

where the gauge-covariant derivative is $D_{\mu} \equiv I \nabla_{\mu}-i T^{a} \mathcal{A}_{\mu}^{a}$, with the generators satisfying $\left[T^{a}, T^{b}\right]=i f^{a b c} T^{c}$ and the inner product taken as $\operatorname{tr}\left(T^{a} T^{b}\right)=\frac{1}{2} \gamma^{a b}$. ${ }^{1}$ We assume that there are $N$ number of Maxwell's fields $F_{\mu \nu}^{k}, k=1,2, \ldots, N$.

Let us take the spacetime to be the $d$-dimensional $p p$ wave geometry with the metric given in the Kerr-Schild form as $g_{\mu \nu}=\eta_{\mu \nu}+2 V \ell_{\mu} \ell_{\nu}$, where $\ell_{\mu}$ is a covariantly constant null vector, and let $A_{\mu}^{k}=\phi^{k} \ell_{\mu}, k=1,2, \ldots, N$ be Abelian and $\mathcal{A}_{\mu}^{a}=\Phi^{a} \ell_{\mu}$ be non-Abelian vector potentials satisfying the properties

$$
\ell^{\mu} \partial_{\mu} \phi^{k}=\ell^{\mu} \partial_{\mu} \Phi^{a}=0 .
$$

Then, one can show that the Einstein tensor reduces to

$$
G_{\mu \nu}=-\ell_{\mu} \ell_{\nu} \dot{\square} V
$$

\footnotetext{
${ }^{1}$ We do not specify the underlying Lie algebra of the nonAbelian theory, but it can be taken to be any non-Abelian Lie algebra.
}

while the Maxwell and Yang-Mills field equations reduce to

$$
\square \phi^{k}=\check{\square} \Phi^{a}=0,
$$

where $\bar{\square} \equiv \eta^{\mu \nu} \partial_{\mu} \partial_{\nu}$. We can now state the first theorem.

Theorem 1: Under the assumptions made above, the field equations (3) reduce to

$$
\bar{\square} V=-\bar{g}^{\mu \nu}\left[\gamma_{a b} \partial_{\mu} \Phi^{a} \partial_{\nu} \Phi^{b}+\sum_{k=1}^{N} \partial_{\mu} \phi^{k} \partial_{\nu} \phi^{k}\right],
$$

whose most general solution is

$V=V_{0}+c_{k} \phi^{k}+\beta_{a} \Phi_{a}-\frac{1}{2} \gamma_{a b} \Phi^{a} \Phi^{b}-\frac{1}{2} \sum_{k=1}^{N} \phi^{k} \phi^{k}$,

where $V_{0}$ is the vacuum solution satisfying $\square V_{0}=0 ; c_{k}$, $\beta^{a}$ are arbitrary constants; and $\phi^{k}$ and $\Phi_{a}$ satisfy Eq. (6).

Given any solution of Eq. (6), and there are many, one can find the corresponding metric via the profile function (8). Observe that if one further takes $\Phi^{a}=t^{a} \phi$ and $\phi^{k}=p^{k} \phi$, where $t^{a}$ and $p^{k}$ are constants, then one only needs to solve a single scalar equation $\square \phi=0$ for $\phi$. Let us note that this solution generalizes the solutions of Refs. [1,2], where $V_{0}=0$ and the gauge fields are treated as test fields that do not change the background geometry, but here we have given the solution of the full coupled system. In the rest of the paper, we do not explicitly consider the Maxwell fields, but we embed them the YangMills fields by enlarging the gauge group. For this purpose, we let the Maxwell fields vanish without losing any generality.

Our next task is to try to generalize this result for the general KSK class, which has been studied in some detail recently in Refs. [6-11]. In generalized Kerr-Schild coordinates, the metric is taken to be $\mathrm{e}^{2}$

$$
g_{\mu \nu}=\bar{g}_{\mu \nu}+2 V \lambda_{\mu} \lambda_{\nu}
$$

where the seed $\bar{g}_{\mu \nu}$ metrics are maximally symmetric. One can show that the following relations hold for the metrics belonging to the KSK class:

$$
\begin{array}{ll}
\lambda^{\mu} \lambda_{\mu}=0, & \nabla_{\mu} \lambda_{\nu} \equiv \xi_{(\mu} \lambda_{\nu)}, \\
\xi_{\mu} \lambda^{\mu}=0, & \lambda^{\mu} \partial_{\mu} V=0 .
\end{array}
$$

The first property is the usual nullity condition of the vector, while the second and the third ones guarantee that the $\lambda$ vector is geodesic, $\lambda^{\mu} \nabla_{\mu} \lambda_{\nu}=0$. These three conditions define the KSK class of metrics. The last property is

\footnotetext{
${ }^{2}$ We use the vector $\lambda_{\mu}$ for this case instead of the previous $\ell_{\mu}$, as we shall reserve $\ell$ for the AdS radius.
} 
required for further simplifications, such as the linear dependence of the mixed Einstein tensor on $V$. For more on this point in the context of Kerr-Schild metrics, see Ref. [12] for the flat seed and Ref. [13] for the generalized cases. For this class of metrics, the traceless Ricci tensor, $S_{\mu \nu} \equiv R_{\mu \nu}-\frac{R}{d} g_{\mu \nu}$, can be computed to yield

$$
S_{\mu \nu}=\rho \lambda_{\mu} \lambda_{\nu}
$$

where the scalar function $\rho$ is found to be linear in $V$, which reads explicitly

$\rho=-\left(\bar{\square}+2 \xi^{\mu} \partial_{\mu}+\frac{1}{2} \xi^{\mu} \xi_{\mu}-\frac{2(d-2)}{\ell^{2}}\right) V \equiv-\mathcal{Q}_{1} V$.

We define the operator $\mathcal{Q}_{1}$ in the second equality. The Weyl tensor, $C_{\mu \alpha \nu \beta}$, can be found to be [8]

$$
C_{\mu \alpha \nu \beta}=4 \lambda_{[\mu} \Omega_{\alpha][\beta} \lambda_{\nu]},
$$

where the square brackets denote antisymmetrization with a $1 / 2$ factor and the symmetric tensor $\Omega_{\alpha \beta}$ is a rather nontrivial object, but it is still linear in $V$ and can be compactly written as

$$
\begin{aligned}
\Omega_{\alpha \beta} \equiv & -\left[\nabla_{\alpha} \partial_{\beta}+\xi_{(\alpha} \partial_{\beta)}+\frac{1}{2} \xi_{\alpha} \xi_{\beta}\right. \\
& \left.-\frac{1}{d-2} g_{\alpha \beta}\left(\mathcal{Q}_{1}+\frac{2(d-2)}{\ell^{2}}\right)\right] V .
\end{aligned}
$$

From the Weyl tensor and the traceless Ricci tensor given here, one can compute the needed curvature invariants for these metrics. As two examples of the KSK class, let us give the AdS plane and the AdS spherical wave metrics, which read as follows:

AdS plane wave metrics:

$$
\begin{aligned}
d s^{2}= & \frac{\ell^{2}}{z^{2}}\left(2 d u d v+\sum_{m=1}^{d-3}\left(d x^{m}\right)^{2}+d z^{2}\right) \\
& +2 V\left(u, x^{m}, z\right) d u^{2}
\end{aligned}
$$

where $z=x^{d-1}, \lambda_{\mu} d x^{\mu}=d u$, and $\xi_{\mu} d x^{\mu}=\frac{2}{z} d z$.

AdS spherical wave metrics:

$d s^{2}=\frac{\ell^{2}}{z^{2}}\left(-d t^{2}+\sum_{m=1}^{d-1}\left(d x^{m}\right)^{2}\right)+2 V\left(t, x^{m}, z\right) d u^{2}$,

where

$$
\begin{aligned}
& \lambda_{\mu} d x^{\mu}=d t+\frac{1}{r} \vec{x} \cdot d \vec{x} \\
& \xi_{\mu} d x^{\mu}=-\frac{1}{r} \lambda_{\mu} d x^{\mu}+\frac{2}{r} d t+\frac{2}{z} d z
\end{aligned}
$$

Here $r^{2}=\sum_{m=1}^{d-1}\left(x^{m}\right)^{2}$. The function $V$ satisfies the constraint $\lambda^{\mu} \partial_{\mu} V=0$. In Ref. [8], we showed that the AdS plane wave and the $p p$-wave metrics, and more generally all KSK metrics, are universal in the sense that they solve all metric-based gravity equations with only slight changes in the parameters, such as the cosmological constant. Different seed metrics $\left(\bar{g}_{\mu \nu}\right)$ lead to different spacetimes: it is the flat Minkowski metric for the $p p$ waves, it is the AdS spacetime for the AdS plane and the AdS spherical waves, and it is the de Sitter spacetime for the dS hyperbolic wave. After this brief recap of the KSK metrics, we can state our second theorem.

Theorem 2: $\quad$ Let the spacetime be the $d$-dimensional KSK geometry with the metric $g_{\mu \nu}=\bar{g}_{\mu \nu}+2 V \lambda_{\mu} \lambda_{\nu}$, and let $\mathcal{A}_{\mu}^{a}=\Phi^{a} \lambda_{\mu}$ be a non-Abelian vector potential, satisfying the property

$$
\lambda^{\mu} \partial_{\mu} \Phi^{a}=0
$$

Then the Einstein Maxwell, Yang-Mills, null dust field equations with cosmological constant

$$
\begin{aligned}
G_{\mu \nu} & =\gamma_{a b} \mathcal{F}^{a \alpha}{ }_{\mu} \mathcal{F}_{\alpha \nu}^{b}-\frac{1}{4} \mathcal{F}^{2} g_{\mu \nu}-\Lambda g_{\mu \nu}+\varepsilon u_{\mu} u_{\nu}, \\
\left(D_{\mu} \mathcal{F}^{\mu \nu}\right)^{a} & =0, \quad \nabla^{\mu}\left(\varepsilon u_{\mu} u_{\nu}\right)=0
\end{aligned}
$$

have the solution

$$
\begin{aligned}
V & =\beta_{a} \Phi_{a}-\frac{1}{2} \gamma_{a b} \Phi^{a} \Phi^{b} \\
\varepsilon & =\left(\xi^{\mu} \partial_{\mu}+\frac{1}{2} \xi^{\mu} \xi_{\mu}-\frac{2(d-2)}{\ell^{2}}\right)\left(\frac{1}{2} \gamma_{a b} \Phi^{a} \Phi^{b}+\beta_{a} \Phi^{a}\right) \\
\Lambda & =-\frac{(d-1)(d-2)}{2 \ell^{2}} \\
u_{\mu} & =\lambda_{\mu} .
\end{aligned}
$$

The Einstein tensor takes the form

$$
G_{\mu \nu}=-\lambda_{\mu} \lambda_{\nu} \mathcal{Q}_{1} V+\frac{(d-1)(d-2)}{2 \ell^{2}} g_{\mu \nu},
$$

while the Yang-Mills equation reduces to

$$
\mathcal{Q}_{2} \Phi^{a}=0
$$

where $\mathcal{Q}_{2} \equiv \dot{\square}+\xi^{\mu} \partial_{\mu}$.

The proof of this theorem is as follows: the field strength of the Yang-Mills fields can be computed to be

$$
\mathcal{F}_{\mu \nu}^{a}=\partial_{\mu} \Phi^{a} \lambda_{\nu}-\partial_{\nu} \Phi^{a} \lambda_{\mu},
$$

whose nonlinear part vanishes. Using the assumption in Eq. (18), one finds 


$$
\nabla^{\mu} \mathcal{F}_{\mu \nu}^{a}=\left(\bar{\square}+\xi^{\alpha} \partial_{\alpha}\right) \Phi^{a} \lambda_{\nu}=0,
$$

which then leads to

$$
\mathcal{Q}_{2} \Phi^{a}=0
$$

The energy-momentum tensor of the gauge field becomes

$$
T_{\mu \nu}^{\mathrm{YM}}=\bar{g}^{\alpha \beta} \gamma_{a b} \partial_{\alpha} \Phi^{a} \partial_{\beta} \Phi^{b} \lambda_{\mu} \lambda_{\nu} .
$$

Then the field equations (19) reduce to

$$
\begin{aligned}
-\mathcal{Q}_{1} V & =\bar{g}^{\alpha \beta} \gamma_{a b} \partial_{\alpha} \Phi^{a} \partial_{\beta} \Phi^{b}+\varepsilon, \\
\Lambda & =-\frac{(d-1)(d-2)}{2 \ell^{2}} .
\end{aligned}
$$

Moreover, one can show that

$$
\mathcal{Q}_{2}\left(\frac{1}{2} \gamma_{a b} \Phi^{a} \Phi^{b}\right)=\bar{g}^{\alpha \beta} \gamma_{a b} \partial_{\alpha} \Phi^{a} \partial_{\beta} \Phi^{b}
$$

Hence, one obtains

$$
-\mathcal{Q}_{2}\left(V+\frac{1}{2} \gamma_{a b} \Phi^{a} \Phi^{b}\right)=\left(\xi^{\mu} \partial_{\mu}+\frac{1}{2} \xi^{\mu} \xi_{\mu}-\frac{2(d-2)}{\ell^{2}}\right) V+\varepsilon .
$$

Assuming that both sides of Eq. (29) vanish, one then obtains Eq. (20). Note that the solution of a single equation $\mathcal{Q}_{2} \Phi^{a}=0$ solves all the Einstein-Yang-Mills and null dust field equations identically. Ignoring the quadratic terms in $V$, we obtain solutions of the Einstein field equations where the Yang-Mills field is a test field. The vanishing of the vector $\xi_{\mu}$ means that the vector $\lambda_{\mu}$ becomes a covariantly constant vector field. In a spacetime with such a vector field, the cosmological constant vanishes identically, and the metric reduces to the $p p$-wave metric. Then, for vanishing $\xi$, the null dust also vanishes, i.e., $\varepsilon=0$, then Theorem 2 reduces to Theorem 1, and hence the proof of Theorem 1 also follows.

For this brief part, let us assume that we have a single Maxwell field and a non-Abelian gauge field. Then, in Theorem 1, it is possible to introduce coupled equations between $\phi$ and $\Phi^{a}$. Let the field equations be

$$
D^{\mu} \mathcal{F}_{\mu \nu}^{a}=\mathcal{J}_{\nu}^{a}, \quad \nabla^{\mu} F_{\mu \nu}=j_{\nu} .
$$

Then, the covariant conservation yields

$$
\nabla^{\mu} G_{\mu \nu}=\gamma_{a b} \mathcal{J}^{a \alpha} \quad \mathcal{F}_{\alpha \nu}^{b}+j^{\alpha} F_{\alpha \nu}=0 .
$$

The right-hand side vanishes identically, since $\mathcal{J}_{\nu}^{a}=$ $\lambda_{\nu} \square \Phi^{a}$ and $j_{\nu}=\lambda_{\nu} \square \phi$. Hence, the Einstein equations in Eq. (3) reduce to

$$
-\square V=\bar{g}^{\alpha \beta} \gamma_{a b} \partial_{\alpha} \Phi^{a} \partial_{\beta} \Phi^{b}+\bar{g}^{\alpha \beta} \partial_{\alpha} \phi \partial_{\beta} \phi,
$$

which is equivalent to the following:

$$
-\bar{\square}\left(V+\frac{1}{2} \gamma_{a b} \Phi^{a} \Phi^{b}+\frac{1}{2} \phi^{2}\right)=-\gamma_{a b} \Phi^{a} \bar{\square} \Phi^{b}-\phi \bar{\square} \phi .
$$

Hence, we can let

$$
V=c \phi+\beta_{a} \Phi^{a}-\frac{1}{2} \gamma_{a b} \Phi^{a} \Phi^{b}-\frac{1}{2} \phi^{2}
$$

and

$$
\begin{aligned}
\square \Phi^{a} & =\phi f^{a}\left(\phi, \Phi^{a}, \partial \phi, \partial \Phi^{a}\right), \\
\square & =-\gamma_{a b} \Phi^{b} f^{a}\left(\phi, \Phi^{a}, \partial \phi, \partial \Phi^{a}\right),
\end{aligned}
$$

where $f^{a}\left(\phi, \Phi^{a}, \partial \phi, \partial \Phi^{a}\right)$ is an arbitrary function of its arguments. Hence, we obtain a coupled system of nonlinear equations for $\phi$ and $\Phi^{a}$. We get a rather simple example by letting $\Phi^{a}=c^{a} \psi$ and $f^{a}=\kappa c^{a}$; then

$$
\square \phi=-\kappa c^{2} \psi, \quad \square \psi=-\kappa \phi,
$$

where $c^{2}=c^{a} c^{b} \gamma_{a b}$. These equations can decoupled as

$$
\bar{\square}^{2} \phi=-m^{2} \phi, \quad \square^{2} \psi=-m^{2} \psi,
$$

where $m^{2}=\kappa^{2} c^{2}$. Similar extension can be made in Theorem 2 as well.

\section{CONCLUSIONS AND FURTHER DISCUSSIONS}

We have studied the $p p$-wave and Kerr-Schild-Kundt geometries as examples of the classical double copy correspondence in the coupled Einstein-Yang-Mills system. For the $p p$-wave case, the metric profile function $(V)$ is given as a quadratic and linear function of the scalar fields defining the Yang-Mills fields as (taking $V_{0}=0$ )

$$
V=\beta_{a} \Phi_{a}-\frac{1}{2} \gamma_{a b} \Phi^{a} \Phi^{b},
$$

which nicely fits in the double copy notion, as gravity is basically the "square of the gauge theory." In the general KSK case, for the double copy correspondence to work, we have shown that one also needs a null dust, a fact which somewhat complicates the correspondence. As a further extension, one might wonder how far one can go if the condition on the nullity of the Kerr-Schild vector field is relaxed. For this purpose, below is a a brief account of an attempt in such metrics. 
Let $\left(g_{\mu \nu}, M\right)$ be a $d$-dimensional spacetime geometry with the metric

$$
g_{\mu \nu}=h_{\mu \nu}-u_{\mu} u_{\nu},
$$

where $u_{\mu}$ is a unit timelike vector field and $h_{\mu \nu}$ is a degenerate matrix of rank $d-1$. We let $u^{\mu}=-\frac{1}{u_{0}} \delta_{0}^{\mu}$ and $u^{\mu} h_{\mu \nu}=0$. The determinant of the metric is $g=-u_{0}^{2}$. We call such a spacetime metric a "Gödel-type metric" $[14,15]$. Here, for a simple construction, we will assume that $u_{0}$ is a nonzero constant and $u_{\mu}$ is a Killing vector field. We assume also that $\partial_{0} u_{\alpha}=0$. With this information, one can find the field strength

$$
f_{\mu \nu} \equiv \nabla_{\mu} u_{\nu}-\nabla_{\nu} u_{\mu}=2 \nabla_{\mu} u_{\nu}, \quad f^{2} \equiv f_{\alpha \beta} f^{\alpha \beta},
$$

and the Einstein equations as

$$
G_{\mu \nu}=\frac{1}{2} T_{\mu \nu}+\frac{1}{4} f^{2} u_{\mu} u_{\nu},
$$

where $T_{\mu \nu}$ is the Maxwell energy-momentum tensor. We have constructed a metric which satisfies the EinsteinMaxwell dust field equations identically provided that the vector field $u_{\mu}$ satisfies the source-free Maxwell equation

$$
\partial_{\alpha} f^{\alpha}{ }_{\mu}=0 .
$$

Observe that the partial derivative appears in this expression, but, under the assumptions made so far, one can show that the last equation is equivalent to the following equation:

$$
\nabla_{\alpha} f_{\mu}^{\alpha}=\frac{1}{2} f^{2} u_{\mu}
$$

or

$$
\left(\square-\frac{1}{4} f^{2}\right) u_{\mu}=0
$$

A simpler version of this equation is $\partial_{i} f_{i j}=0$. All the above equations on the vector $u_{\alpha}$ can be simplified further. Since $u_{0}$ is assumed to be constant, then $\vec{u}$ satisfies the linear equation

$$
\nabla^{2} \vec{u}-\vec{\nabla}(\vec{\nabla} \cdot \vec{u})=0
$$

Hence, any solution of the last equation also solves Einstein-Yang-Mills field equations identically where $A_{\mu}^{a}=$ $c^{a} u_{\mu}$ and $g_{\mu \nu}=h_{\mu \nu}-u_{\mu} u_{\nu}$. When $u_{0}$ is not a constant, then the metric can be extended further to a scalar (dilaton) field. Gödel-type metrics can be used in solving the EinstenYang-Mills dilaton 3-form field equations [15] from a single vector equation. These metrics deserve a separate discussion, which we shall give elsewhere.
[1] R. Monteiro, D. O'Connell, and C. D. White, Black holes and the double copy, J. High Energy Phys. 12 (2014) 056.

[2] M. Carrillo-Gonzalez, R. Penco, and M. Trodden, The classical double copy in maximally symmetric spacetimes, J. High Energy Phys. 04 (2018) 028.

[3] Z. Bern, J. J. M. Carrasco, and H. Johansson, New relations for gauge-theory amplitudes, Phys. Rev. D 78, 085011 (2008).

[4] A. Luna Godoy, Ph.D. thesis, University of Glasgow, http:// theses.gla.ac.uk/8716/.

[5] K. Lee, Kerr-Schild double field theory and classical double copy, J. High Energy Phys. 10 (2018) 027.

[6] I. Gullu, M. Gurses, T. C. Sisman, and B. Tekin, AdS waves as exact solutions to quadratic gravity, Phys. Rev. D 83, 084015 (2011).

[7] M. Gurses, T. C. Sisman, B. Tekin, M. Gurses, T. C. Sisman, and B. Tekin, Some exact solutions of all $f\left(R_{\mu \nu}\right)$ theories in three dimensions, Phys. Rev. D 86, 024001 (2012); New exact solutions of quadratic curvature gravity, Phys. Rev. D 86, 024009 (2012).

[8] M. Gurses, T. C. Sisman, B. Tekin, and S. Hervik, AdSWave Solutions of $f$ (Riemann) Theories, Phys. Rev. Lett. 111, 101101 (2013).
[9] M. Gurses, T. C. Sisman, and B. Tekin, AdS-plane wave and $p p$-wave solutions of generic gravity theories, Phys. Rev. D 90, 124005 (2014); Gravity waves in three dimensions, Phys. Rev. D 92, 084016 (2015).

[10] M. Gurses, T.C. Sisman, and B. Tekin, From smooth curves to universal metrics, Phys. Rev. D 94, 044042 (2016).

[11] M. Gurses, T. C. Sisman, and B. Tekin, Kerr-Schild-Kundt metrics are universal, Classical Quantum Gravity 34, 075003 (2017).

[12] M. Gurses and F. Gursey, Lorentz covariant treatment of the Kerr-Schild metric, J. Math. Phys. (N.Y.) 16, 2385 (1975).

[13] T. Dereli and M. Gurses, The generalized Kerr-Schild transform in eleven-dimensional supergravity, Phys. Lett. B 171, 209 (1986).

[14] M. Gurses, A. Karasu, and O. Sarioglu, Gödel-type metrics in various dimensions, Classical Quantum Gravity 22, 1527 (2005).

[15] M. Gurses and O. Sarioglu, Gödel-type metrics in various dimensions II: Inclusion of a dilaton field, Classical Quantum Gravity 22, 4699 (2005). 